\title{
THE RESEARCH OF FUZZY PID CONTROL STRATEGY BASED ON NEURAL NETWORK IN THE TENSION SYSTEM
}

\author{
Yongyi He, Shuai Guo, Qihong Zhou \\ School of Mechanical Engineering and Automation, Shanghai University, Shanghai,China. \\ Email: richie_gs@sina.com.
}

\begin{abstract}
Based on back-propagation (BP) arithmetic, a neural network Fuzzy PID control strategy is presented for the tension systems, the parameters of which are of deep coupling, nonlinearity, time-variability, and indeterminacy. In this control strategy, the parameters such as proportion, integration and differentiation of PID controller are adjusted on-line through self-learning of a network based on classical PID control algorithm. The decoupling control is carried out in the process. The performance of control strategy has been performed on the tension tested-bed under the varying load conditions. The experimental results show that the almost dynamic decoupling and completely static decoupling are obtained.
\end{abstract}

Key words: Tension System; Decoupling Control; Neural Network

\section{INTRODUCTION}

Tension control technology is one of the common technologies exclusively used in the engineering industry such as wire saw, IC manufacture, printing equipment, fibre wind and etc. Along with the modern wind machine developing to higher speed and more precision, tension control technology becomes more important. Due to the fact that tension system can be affected by many factors, its structures are complex and its parameters are of deep coupling, nonlinearity, time-variability, and indeterminacy. Traditional control method is hard to carry out effective control. Some papers ${ }^{[1,2]}$ proposed the fixed-gain and variable-gain PID control of web tension in the winding section. But the effect is not satisfactory. Other engineers have tried to derive a mathematical model of

Please use the following format when citing this chapter:

He, Yongyi, Guo, Shuai, Zhou, Qihong, 2006, in International Federation for Information Processing (IFIP), Volume 207, Knowledge Enterprise: Intelligent Strategies In Product Design, Manufacturing, and Management, eds. K. Wang, Kovacs G., Wozny M., Fang M., (Boston: Springer), pp. 550-556. 
the tension system used in a model-based controller ${ }^{[3]}$, which results a fifthdegree differential equation described the mechanical behavior. In theory, it could work, but in fact it does not. For the complex electromchanical tension control system there are still many problems on its basal theoretics, check and measure technology and control technology to be solved. It is one of the problems of great significance for the technician to study.

Taking "Fuzzy logic PID decoupling control strategies based on neural network" as a main topic, this paper established a new kind of MIMO fuzzy PID decoupling controller based on BP neural network for the complex electromchanical tension systems. Compared with other control strategies, the structure and learning algorithm of the strategies presented in this paper are relatively simple and physical meanings of neuron in input and output layer are determinate. The parameters of PID controller on-line are adjusted according to a certain control law and through self-study of neural network. Then the manipulated variable gotten through classical PID control algorithm is employed to the control and the decoupling is realized in this course while it need not use the given sampling signal to study offline. By the way the structure of controller is defined in the light of the number of input and output variable. The structure of control object needs to be known in advance. The weight coefficient of network connection and values of parameters in PID controller can be taken by course of fuzzy logic control.

\section{STRUCTURE OF NEURAL NETWORK FUZZY LOGIC PID CONTROLLER OF TENSION CONTROL SYSTEM}

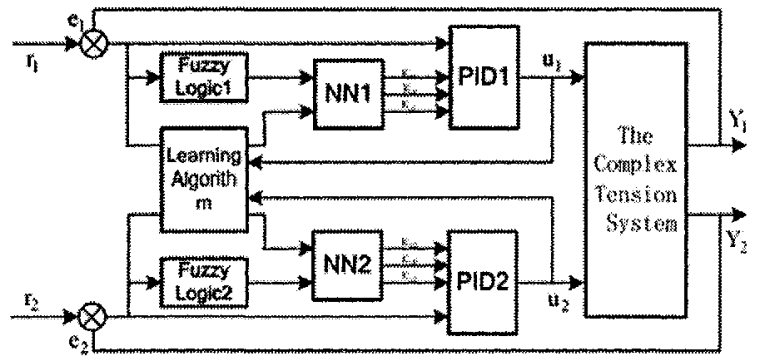

Fig. 1 Basic Tension Controller Architect ${ }^{[4]}$

Tension systems are couple input and couple output system. The structure of tension control system is made up of neural network NN and classical fuzzy logic PID controller. Structure diagram is shown in figure 1, in which $r_{1}$ and $y_{1}$ correspond with the given value and output value of 
practical system tension respectively, while $r_{2}$ and $y_{2}$ correspond with the given value and output value of system speed, respectively. Close loop control is carried out to control object by classical PID controller. While the three parameters $K_{P}, K_{i}$, and $K_{d}$ are adjusted by neural network, they includes learning algorithm, fuzzy logic and neural network NN. Parameters of PID controller can be adjusted on line through the learning algorithm based on the operation state. So performance figures can get to optimization.

Neural network NN is a sub-network including three layers. The number of input layer nodes is $M=3$, hidden layer nodes $Q=6$, output layer nodes $L$ $=3$. The three nodes in input layer accept error $E(k)$, error sum $\sum E(k)$ and error increment $\Delta \mathrm{E}(\mathrm{k})$ respectively; The three nodes in output layer correspond with the parameters of PID controller, $K_{P}, K_{i}$, and $K_{d}$. Synthesis of all the sub-network $\mathrm{N}=2$ is carried out in control study algorithm. And MIMO control system is formed through combining it with fuzzy logic PID control.

\section{DESIGN SCHEME OF FUZZY PID DECOUPLING CONTROLLER BASED ON NEURAL NETWORK FOR THE TENSION SYSTEM}

The computational method of fuzzy PID controller based on the neural network is made of three sectors: fuzzy logic, classical PID control algorithm and multi-variable learning algorithm based on neural network.

Fuzzy logic algorithm: As fuzzy logic tuning mechanism is used for the on-line adjusted BP neural network, we use standard terminology from fuzzy set theory. Associated with the map $F$ is a collection of linguistic values, each membership function is a map from the real line to the interval $[0,1]$. The membership functions used are of the triangular type. The height of the membership functions in this case is 1 , which occurs at the points $-1,-0.66,-$ $0.34,0,0.34,0.66,1$ respectively.

The realization of the function $\mathrm{r}(\mathrm{k})=\mathrm{F}[\mathrm{e}(\mathrm{k}), \Delta \mathrm{e}(\mathrm{k})]$, based on the standard fuzzy method, consists of three stages: fuzzification, Fuzzy rule inference, and defuzzification. We describe each of these stages in turn.

Fuzzification: The process of fuzzification transforms the inputs $\mathrm{e}(\mathrm{k})$ and $\Delta \mathrm{e}(\mathrm{k})$ into the setting of linguistic values. This consists of scaling the inputs $\mathrm{e}(\mathrm{k})$ and $\Delta \mathrm{e}(\mathrm{k})$ appropriately and then converts them into fuzzy sets. We use the symbol $\mathrm{C}_{\mathrm{e}}$ for the scaling constant for the inpute(k), and the symbol $_{\Delta \mathrm{e}}$ for the scaling constant for the input $\Delta \mathrm{e}(\mathrm{k})$. For each linguistic 
value $l \in L$, we assign a pair of numbers $n_{e}(l)$ and $n_{\Delta c}(1)$ to the inputs $e(k)$ and $\Delta \mathrm{e}(\mathrm{k})$ via the associated membership function $\mathrm{u}_{1}$, by :

$$
\begin{gathered}
\mathrm{n}_{\mathrm{e}}(\mathrm{l})=\mathrm{u}_{1}\left(\mathrm{C}_{\mathrm{e}} \mathrm{e}(\mathrm{k})\right) \\
\mathrm{n}_{\Delta \mathrm{e}}(\mathrm{l})=\mathrm{u}_{1}\left(\mathrm{C}_{\Delta \mathrm{e}} \Delta \mathrm{e}(\mathrm{k})\right)
\end{gathered}
$$

The numbers $\mathrm{n}_{\mathrm{e}}(\mathrm{l})$ and $\mathrm{n}_{\Delta \mathrm{e}}(1), l \in \mathrm{L}$ are used in the computation of $\mathrm{F}[\mathrm{e}(\mathrm{k}), \Delta \mathrm{e}(\mathrm{k})]$, which we will describe when discussing the defuzzification stage.

Fuzzy rule Inference: Associated with the decision making process is a set of fuzzy rules $R=\{R 1, R 2, R 3, \cdots R r\}$ where $r$ is the total number of rules. Our rules derived by using a combination of experience "trial and error" and our knowledge of the response of the system. Suppose that the command signal is a constant $y_{m}$, the error $e(k)$ is zero and the change of error $\Delta \mathrm{e}(\mathrm{k})$ is a negative number, which means that the output $e(k)=y_{m}(k)-y_{p}(k)$ is increasing, i.e., heading in the direction of overshoot. To compensate for this, we decrease the command signal. This corresponds to applying a correction term $\mathrm{r}(\mathrm{k})$ which is negative. Hence, we get the rules "if error is zero and change-of-error is negative- medium then output is a negative-big correction term". The other rules are obtained in the similar manner.

Defuzzification: The defuzzification process maps the result of the fuzzy logic rule stage to a real number output $\mathrm{F}[\mathrm{e}(\mathrm{k}), \Delta \mathrm{e}(\mathrm{k})]$. We use the height defuzzification method, which is simple to implement and comes to relatively good results. To describe this method, let $p(l), l \in L$, be the location of peak of the membership function $\mu$ l. Recall that locations are given by $\mathrm{p}(\mathrm{NB})=-1, \mathrm{p}(\mathrm{NM})=-0.66, \mathrm{p}(\mathrm{NS})=-0.34, \mathrm{p}(\mathrm{ZO})=0$, $\mathrm{p}(\mathrm{PS})=0.34, \mathrm{p}(\mathrm{PM})=0.66, \mathrm{p}(\mathrm{PB})=1$, define:

$$
a_{i}=n_{e}\left(l_{i}^{e}\right) \wedge n_{\Delta e}\left(l_{i}^{\Delta e}\right)
$$

Where $\Delta$ represents the $\min$ operator; Then, the output of the defizzification process is given by

$$
\mathrm{F}[\mathrm{e}(\mathrm{k}), \Delta \mathrm{e}(\mathrm{k})]=\mathrm{C}_{\mathrm{F}} \frac{\sum_{i=1}^{27} p\left(l_{i}\right) a_{i}}{\sum_{i=1}^{27} a_{i}}
$$


Where $\mathrm{C}_{\mathrm{F}}$ represents the scaling factor, this completes our description of the term $r(k)=F[e(k), \Delta e(k)]$.

Multi-variable study algorithm based on BP neural network resembles other BP algorithm ${ }^{[5]}$. It is made up of two sectors: forward and back propagation algorithm. The three neurons in input layer correspond with the parameters of PID controller $\mathrm{K}_{\mathrm{p}}, \mathrm{K}_{\mathrm{i}}$, and $\mathrm{K}_{\mathrm{d}}$ respectively and they are not to be negative number. So activation function in output layer neural network can take sigmoid function of positive and negative symmetric. Alphabet $p$ stands for sequence number of sub-network.

Neural network algorithm: In any sampling time the computation formula of forward propagation algorithm of neural network is given as following:

Input of input layer of neural network $\mathrm{NN}$ is:

$$
\left\{\begin{array}{l}
x_{p 1}^{\prime}(k)=e_{p}(k) \\
x_{p 2}^{\prime}(k)=\sum_{i=0}^{k} e_{p}(i) \\
x_{p 3}^{\prime}(k)=e_{p}(k)-e_{p}(k-1)
\end{array}\right.
$$

Input and output of hidden layer is:

$$
\left\{\begin{array}{l}
\text { net }_{p i}^{\prime \prime}(k)=\sum_{j=1}^{M} \omega_{p i j}^{\prime \prime} x_{p j}^{\prime}(k)-\theta_{p i}^{\prime \prime} \\
x_{p i}^{\prime \prime}(k)=f\left[\text { net }_{p i}^{\prime \prime}(k)\right]
\end{array}\right.
$$

In expression (6), $\mathrm{M}$ is neuron number of input layer, $\omega_{\mathrm{pij}}^{\prime \prime}$ is connection weight coefficient in sub-network of hidden layer; $\theta_{\mathrm{pi}}^{\prime \prime}$ is threshold value of neuron in hidden layer; $f(\bullet)$ is activation function in hidden layer $\mathrm{f}(\bullet)=\tanh (\mathrm{x})$;

Input and output of output layer is:

$$
\left\{\begin{array}{l}
\text { net }_{p i}^{\prime \prime}(k)=\sum_{j=1}^{Q} \omega_{p i j}^{\prime \prime} x_{p j}^{\prime}(k)-\theta_{p i}^{\prime \prime} \\
x_{p i}^{\prime \prime}(k)=g\left[\text { net }_{p i}^{\prime \prime}(k)\right]
\end{array}\right.
$$

In expression (7), $Q$ is neuron number of hidden layer; $\omega_{\mathrm{pij}}^{\prime \prime}$ is connection weight coefficient in output layer; $\theta_{\mathrm{pi}}^{\prime \prime}$ is corresponding threshold value; $\mathrm{g}(\bullet)$ is activation function in output layer $\mathrm{f}(\bullet)=0.5[1+\tanh (\mathrm{x})]$. 


\section{DESIGN OF TENSION CONTROLLER BASED ON DSP}

In order to proof the performance of the tension controller with fuzzy PID decoupling control strategy based on neural network, we designed the tension control board based on DSP controller. DSP controllers available today are able to perform the computation for high performance DMC structures for different motor technologies and motion control configurations.

The core part is based on a novel 16-bit TMS320LF2407 DSP module. It has a 30MIP's 16 fixed point DSP core with up to $30 \mathrm{MHz}$ CPU frequency. It also integrates the following power electronics peripherals-two event manager modules (EVA\&EVB) with two general-purpose timers (GP Timers), three capture units and eight PWM outputs for each module. It also has other features such as a $3.3 \mathrm{~V}$ power supply, a built-in quadrature encoder pulse (QEP) circuit, a high speed A/D converter, as well as a large number of digital I/O. The characteristics mentioned above make it quite flexible especially for digital motion control applications.

In the software design, the real time control is a very serious problem in the tension control system. Now the interrupt technology is very popular in real time control. In general sense, the real time is implemented by interruption of the real time clock. The real time clock is in charge of managing control process, collecting the related signals, inspiring all kinds of control task, and sending the related control signal. So the real time clock is very important in the real time control ${ }^{[6]}$.

The performance test of the tension control board with PID control algorithm based on DSP has been performed on the tension tested-bed under the varying load conditions. Fig. 2 shows the basic system architecture of the tension tested-bed. Fig. 3 shows the plots of tension and speed responses in a speed control mode when there is a change in the speed from 0 to 100 RPS with $25 \%$ parameter error. Fig. 3 (a) shows the case with the conventional PID controller, while Fig. 3 (b) shows the case with proposed fuzzy PID control strategy based on neural network controller. The speed variation in Fig. 3 (a) tells us the imperfect decoupling, and Fig. 3 (b) shows its advantages of higher speed response and stronger robustness.

\section{CONCLUSIONS}

PID decoupling control algorithm based on neural network is proposed 
in this paper. It makes classical PID control law induct in BP neural network. The structure of controller is simple and its principle is definite. So it is easy to be designed and to be carried out. The ability of dealing with dynamic problems is very good. We can control the system under the circumstance of unknown state perfectly and search and correct parameters of the controller on line. Experimental study is carried out on the tension tested-bed under the varying load conditions. Results show that the system has strong robustness, adaptive faculty and good control quality even when the model has changed.

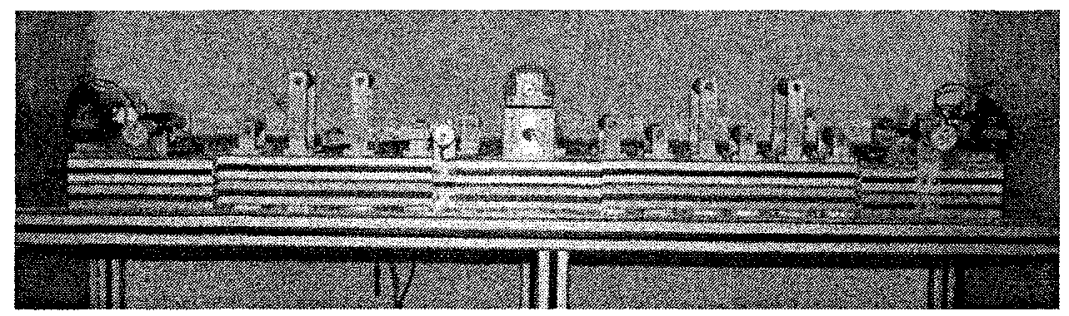

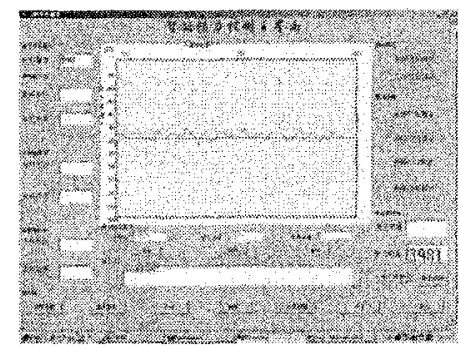

Fig.3a Results of Experiment

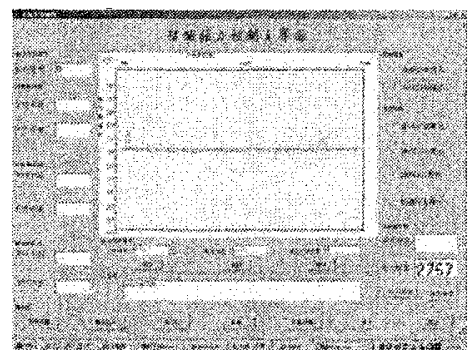

Fig. 3b Results of Experiment

\section{REFERENCE}

1. Y. Hou, Z. Gao, F. Jiang and B. T. Boulter, "Active Disturbance Rejection Control for Web Tension", Decision and Control, 2001. Proceedings of the 40th IEEE Conference, Volume: 5, 4-7 Dec. 2001 Pages: 4974 - 4979 vol.5 Regulation

2. Berger Larhr, Technical Document, November 1998, pp.1-3

3. J. C. Basilio and S. R. Matos, "Design of PI and PID Controllers with Transient Performance Specification", IEEE Transactions on education, VOL.45, NO.4, November 2002, pp.364-366

4. Jong-Hwan Kim, "Fuzzy Precompensation of PID Controllers", Second IEEE Conference on Control Applications, pp.184-186

5. Karl N. Reid, Kee-Hyun Shin, and $\mathrm{Ku}-\mathrm{Chin}$ Lin, "Variable-Gain Control Of Longitudinal Tension In A Web Transport System", AMD-Vol. 149, Web Handling, ASME 1992, pp87-100.

6. TAN shi-zhi, Yang Ru-qing, "Design of Industrial Robotic Controller Based on DSP", Journal of Donghua University(Eng. ED.), VOL.20, No.1, 2003, pp.61-65 